\title{
Fourier Independent Component Analysis of Radar Micro-Doppler features
}

\author{
P. Addabbo ${ }^{1}$, C. Clemente ${ }^{2}$, and S.L. Ullo ${ }^{3}$ \\ ${ }^{1}$ Università Telematica Giustino Fortunato, Benevento-Italy \\ ${ }^{2}$ University of Strathclyde, Glasgow-United Kingdom \\ ${ }^{3}$ Università degli Studi del Sannio, Benevento-Italy
}

\begin{abstract}
The capability of discriminating radar targets exhibiting multiple moving parts has become of great interest for both aerospace and ground-based target recognition and analysis. In particular, helicopters and other targets with rotors, as for instance miniature Unmanned Aerial Vehicles, exhibit peculiar characteristics in the radar return that can be used for their recognition. In this paper a novel algorithm to address the problem of micro-Doppler signature unmixing is proposed, exploiting the signal separation capabilities of the Independent Component Analysis (ICA). The core of the algorithm is represented precisely by the use of the ICA procedure, that has been already proved to be a very effective technique for separating hidden information in mixtures of observations. ICA has been successfully employed in several applications such as wireless communications, radar beamforming, trace-gases unmixing and medical imaging processing. The helicopter's rotor blade signature unmixing from a multi-static radar system is considered as case study and results obtained through the application of ICA to simulated multi-component micro-Doppler signatures show the capability of the proposed approach to successfully accomplish the unmixing operation.
\end{abstract}

Index terms - helicopter classification, micro-Doppler features, Independent Component Analysis (ICA)

\section{INTRODUCTION}

In recent studies the main objectives of micro-Doppler analysis and investigation have been to pursue reliable micro-Doppler signature classification procedures, able of describing and identifying uniquely the target by using a very limited amount of data and observations. Very interesting researches in this sense are presented in [1]-[2] where information on micro-Doppler signatures is extracted from the cadence velocity diagram (CVD) of received data in order to perform target recognition. The approach proposed in [1] aims to classify human activity based on the actual cadence frequencies used as features. In [2] a novel algorithm is presented, based on the application of orthogonal pseudo-Zernike polynomials. Features of micro-Doppler are derived from the pseudo-Zernike moments extracted from the CVDs. The proposed algorithm has shown good properties of invariance with respect to translation and scale dependencies, and high accuracy in classification of real micro-Doppler data in $\mathrm{Ku}$ - and $\mathrm{X}$ - bands. In [3] a modelbased approach exploiting sparsity has been used to recognize helicopters' returns, allowing the target recognition based on the knowledge of the helicopter's rotor characteristics only and no express need of real training radar measurements. The algorithm has been proved to get very good results in almost all the cases of interest. Since in many situations where a target is composed by a main body with other moving parts, rotating or vibrating, such as the case of helicopter blades, the reflected signal is the superposition of all these components, the capability to unmix these returns in either the time, frequency or time-frequency domain would provide a potential benefit for the development of advanced target recognition algorithms based on micro-Doppler.

In this paper an algorithm to pursue this objective is presented exploiting a version of Independent Component Analysis (ICA) applied to the time-frequency representation of the mixed signal. ICA has been proved to be a very effective technique for separating complex-valued signals hidden in mixtures of observations. It has found great utility in several applications where signal unmixing was required, such as wireless communications [4], radar beamforming [5], trace-gases retrieval from hyperspectral data [6], data analysis in magnetic resonance imaging [7] and electroencephalograph [8]. In [9] a method for using complex-valued ICA to radar target detection in sea clutter has been successfully applied, and in [10] spatial and temporal ICA of microDoppler features has been studied by using simulated data from rotating and tumbling objects, but at the best of our knowledge ICA has not yet been applied in the frequency domain to the spectrograms of the signal samples containing micro-Doppler components belonging to moving helicopter blades.

The paper is organized as follows. Section II introduces the signal model of a target with multiple moving parts, in particular the helicopter rotor case from multiple-receivers is assumed as case study. Section III introduces the Fourier ICA method used in this paper. Section IV illustrates the proposed algorithm for signature unmixing. Results on simulated radar data are presented in Section V. Conclusions and future work are reported in Section VI.

\section{SignAl MODEL}

The analyzed scenario is represented by an helicopter observed at the same time in a coherent multistatic-system 
with $M$ receivers illuminating the same surveillance area. An illustration of the scenario is shown in Figure 1.

Without loss of generality the baseband slow-time micro-

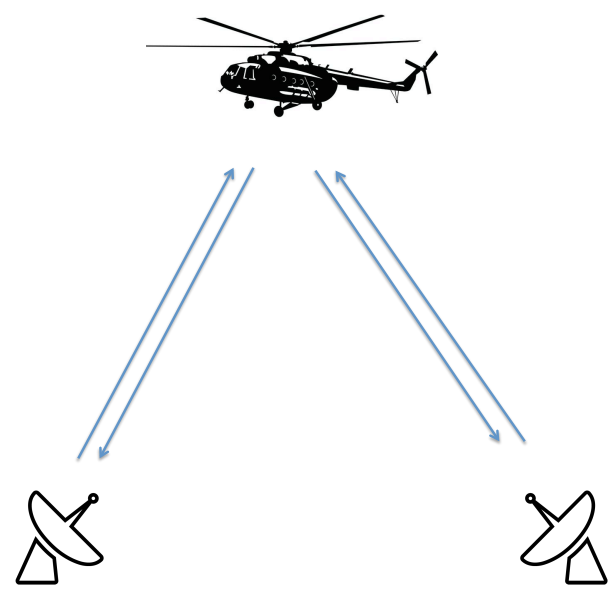

Fig. 1. Observation scenario consisting in a multistatic-system with 2 radar illuminating an helicopter.

Doppler signal received at the $i$-th receiver from the helicopter can be written as [3], [11]:

$$
x_{i}(n)=\sum_{l=0}^{K-1} \sigma_{l, i} \exp ^{-j \frac{2 \pi}{\lambda} \rho \Delta_{i} \cos \left(\omega n T_{s}+\frac{2 \pi}{K}+\theta\right)}
$$

with

$$
\sigma_{l, i}=\operatorname{sinc}\left(\frac{2}{\lambda} \rho \Delta_{i} \cos \left(\omega n T_{s}+\frac{2 \pi}{K} l+\theta\right)\right)
$$

where $i=1, \ldots, M$, represents the index of $i$-th receiver, $K$ is the total number of blades of the rotor, $\sigma_{l, i}$ is the reflectivity of the $l$-th blade seen from the $i$-th receiver, $\lambda$ is the radar carrier wavelength, $\rho$ is the rotor blade length, $\omega$ is the rotor angular velocity, $\theta$ is the rotor initial angle, $T_{s}$ is the sampling period 1 and $\Delta_{i}$ is the scaling factor depending on the specific transmitter-target-receiver geometry.

To better illustrate the nature of the signals at hand the spectrogram is generally used as representation of the microDoppler signatures, obtained through the calculation of the short FFT of the $x_{i}(n)$ samples. Since the $\chi_{i}(\nu, \gamma)$ values are generally complex, the spectrogram is represented on the frequency axis through its square modules $\left|\chi_{i}(\nu, \gamma)\right|^{2}$ in accordance with the equation (3):

$$
\chi_{i}(\nu, \gamma)=\left|\sum_{n=0}^{N-1} x_{i}(n) h(n-\gamma) \exp \left\{-j \frac{2 \pi \nu n}{N}\right\}\right|^{2}
$$

for $\gamma=0, \ldots, \Gamma-1$, and with $\nu$ the normalized frequency and $h(\cdot)$ the smoothing window respectively.

\footnotetext{
${ }^{1}$ In a pulsed radar it is the radar Pulse Repetition Interval
}

As an example the micro-Doppler signature of an oscillating point target observed from 2 different aspect angles is shown in Figure 2, in which it is evident the dependence on the aspect angle affecting the maximum micro-Doppler shift.
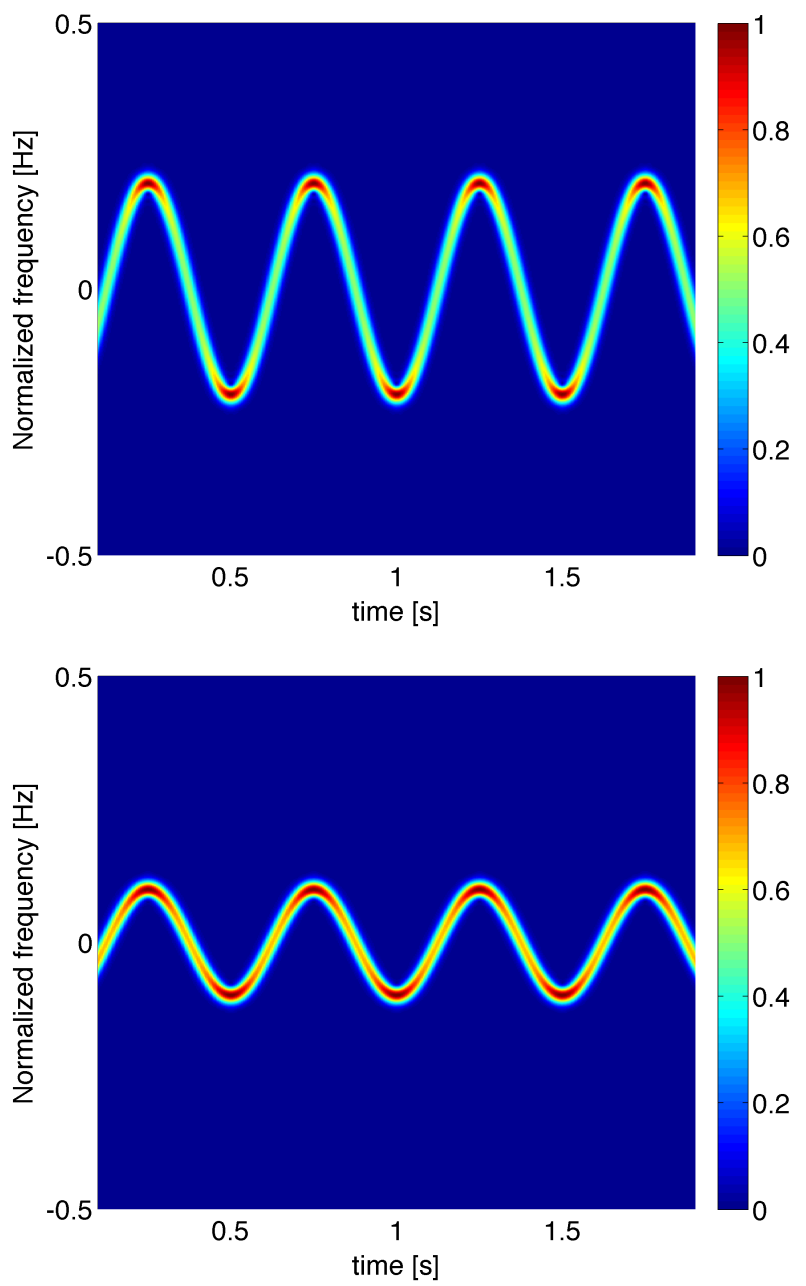

Fig. 2. Micro-Doppler of an oscillating target from 2 different aspect angles.

\section{THE FOURIER ICA MODEL}

Assuming that the micro-Doppler features are statistically independent it is possible to interpret the spectrogram values as observations of a mixing model resolvable with ICA. Specifically, supposing of reshaping the spectrograms in vector form, it is possible to consider the model

$$
\chi=\mathbf{A S}
$$

where:

- $\chi$ is the vector of the $M$ spectrogram observables,

- $\mathbf{S}$ is the vector of the $N$ micro-Doppler features within the spectrograms, with $N \leq M$, 
- A is the $M \times N$ mixing matrix (if $M>N$ a common practice is to reduce the observation dimensionality from $M$ to $N$ ).

With ICA the unmixing is performed in a single step, estimating both micro-Doppler features and mixing matrix components at the same time. Thus, Eq. 4 represents the ICA standard probem model, whose solution is found via a trasformation $\mathbf{W}$, that maximizes the statistical independence of the sources $\mathbf{S}$, on the observations $\chi[12]$

$$
\mathbf{Y}=\mathbf{W} \chi=\mathbf{W A S} .
$$

Note that, if $\mathbf{W}$ is equal to the inverse of the mixing matrix $\mathbf{A}$, the estimate sources $\mathbf{Y}$ will coincide with the sources vector S.

The problem of finding the linear transformation (5) that reaches statistical independence can be organized in two steps: first a whitening operation, to make data uncorrelated, with the same variance, and then a coordinate rotation [13], that preserves whiteness and gets data independence. This means that the transformation matrix is decomposed as $\mathbf{W}=\mathbf{R V}$ where $\mathbf{V}=\boldsymbol{\Lambda}_{\mathbf{X}}{ }^{-1 / 2} \mathbf{U}_{\mathbf{X}}{ }^{T}$ is the whitening matrix, defined through eigenvalues $\Lambda_{\mathbf{X}}$ and eigenvectors $\mathbf{U}_{\mathbf{X}}$ of the covariance matrix of $\mathbf{X}$, and $\mathbf{R}$ is a unitary, rotation matrix that can be found by minimizing a measure of dependence.

A possible measure of statistical dependence among random variables is the mutual information: it is always nonnegative and is zero if and only if all variables are mutually independent. Using the Shannon differential entropy, defined as [14]

$$
\begin{aligned}
\mathrm{H}\left(Y_{1}, \ldots, Y_{N}\right) & = \\
& -\int p\left(y_{1}, \ldots, y_{N}\right) \log \left[p\left(y_{1}, \ldots, y_{N}\right)\right] d y_{1} \ldots d y_{N}
\end{aligned}
$$

the mutual information of the observables can be expressed as [14]

$$
\mathrm{I}\left(Y_{1}, \ldots, Y_{N}\right)=\sum_{n=1}^{N} \mathrm{H}\left(Y_{n}\right)-\mathrm{H}\left(Y_{1}, \ldots, Y_{N}\right) .
$$

It can be shown [12] that minimizing the mutual information with respect to the rotation angles is equivalent to minimizing the sum of the Shannon entropies of the observed random variables.

\section{UNMIXING OF RADAR MICRO-DOPPLER SIGNATURES}

For simplicity we refer here to the case of two receivers, even if it is worth to notice that extension to multiple receivers is possible, but it is out of the scope of this paper.

In the scheme reported in Figure 3, we show the three main steps accomplished to unmix the micro-Doppler features from the received signals.

\section{A. Pre-processing}

Firstly, it is necessary to pre-process the received baseband signals, $x_{1}(n), x_{2}(n)$, as defined in the Equation (1), in order to obtain next two equal sized spectrograms of the observations, on which to apply the ICA technique. This pre-processing step consists substantially of removing the dependence from the geometry scale factor $\Delta_{i}$ in the phase term, that is related to the specific viewing geometry. In order to do that the micro-Doppler bandwidths of the two received signals are estimated to evaluate the re-sampling factor necessary to obtain the scale-invariance of the phase term. Specifically, as outlined in the block scheme of Figure 3 , the second received signal $x_{2}(n)$ is re-sampled, using the factor $N_{d}$ evaluated as the ratio between the estimated bandwidths, thus obtaining the new sequence $x_{2 S}\left(n N_{d}\right)$. This is a necessary step for the successive application of the unmixing via ICA, because different scale factors would have produced an increasing of the unknown sources to be extracted.

\section{B. Spectrogram calculation}

The second step of the procedure consists of the spectrogram calculation, as defined in the Equation (3), for both the received signals, with fixed dimensions, in terms of the number $U$ of FFT bins and the time window size $V$. In Figures 4 and 5 , the spectrograms of the simulated signals received from the first and the second receivers are shown, respectively. In Figure 5, it is also shown the spectrogram of the original signal, at the top, and that of the re-sampled version. It is possible to notice that re-sampling procedure has resulted in a spectral leakage effect, that is then compensated in the third algorithm step using a cascade ICA. The results of spectrogram calculation are the matrices $\chi_{1}$ and $\chi_{2}$, that have been forced to have equal dimensions $U \times V$, to effectively apply the unmixing procedure.

\section{Unmixing}

The last step consists of the unmixing process aiming at the micro-Doppler feature extraction. As pointed out in the step B and shown in the Figures 4 and 5, the re-sampling procedure accomplished in the pre-processing step has resulted into a spectral leakage. In this step we show that this leakage can be efficiently removed via ICA. The unmixing is reached via two ICAs in cascade. The first unmixing is obtained by reshaping the two matrices $\chi_{1}$ and $\chi_{2}$ into vectors of length $1 \times U V$ and arranging them into a matrix $\chi$ of dimension $2 \times U V$ so that:

$$
\chi=\mathbf{A Z}
$$

where $\mathbf{A}$ represents the $2 \times 2$ mixing matrix and $\mathbf{Z}$ is the $2 \times U V$ independent sources matrix. Therefore, the problem can be splitted into two steps: first a whitening operation, to make data uncorrelated and with the same variance, and then a coordinate rotation, that preserves whiteness and gets 


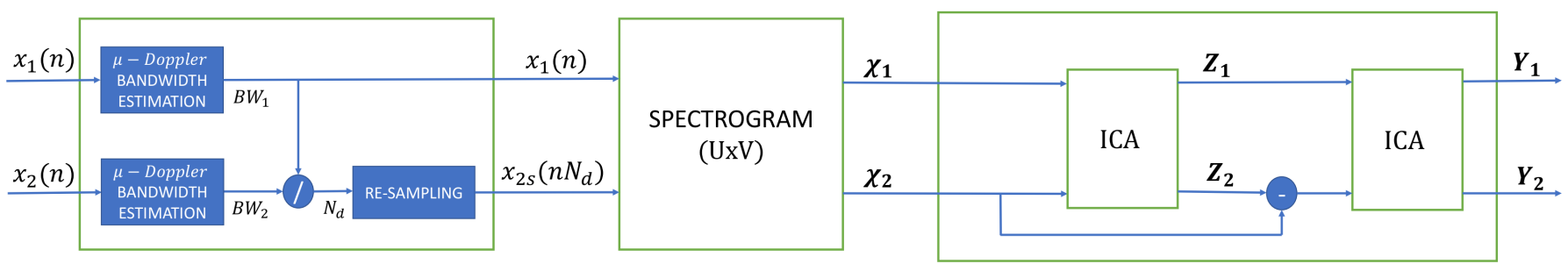

Fig. 3. Block diagram of the proposed unmixing algorithm.

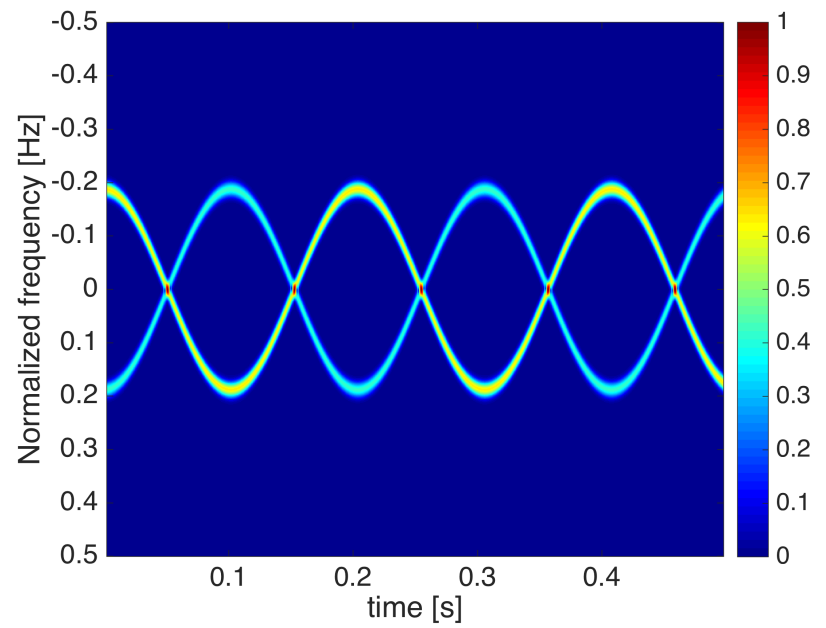

Fig. 4. Spectrogram of the signal received at the first receiver.

statistical independence of data [15], as explained in detail in Section III.

As a standard ICA problem, the solution is found via a matrix transformation $\mathbf{W}_{\mathbf{1}}$, that maximizes the statistical independence of the sources on the observations:

$$
\mathbf{Z}=\mathbf{W}_{\mathbf{1}} \chi \text {. }
$$

The first ICA is thus able to discriminate between the mixed micro-Doppler features and the spectral leakage effect, namely the extracted independent source matrices components, $\mathbf{Z}_{\mathbf{1}}$ and $\mathbf{Z}_{2}$.

The second application of ICA uses as input the first extracted source $\mathbf{Z}_{1}$ and the spectral leakage purified component $\chi_{2}-\mathbf{Z}_{2}$. The micro-Doppler features extraction is finally achieved via a second matrix transformation $\mathbf{W}_{2}$ as before:

$$
\mathbf{Y}=\mathbf{W}_{\mathbf{2}} \mathbf{Z} \text {. }
$$

This second ICA application finally permits to unmix the two sources as rows of $\mathbf{Y}$.

\section{RESULTS ON SIMULATED RADAR DATA}

In this section the results obtained applying the ICA unmixing procedure to simulated radar micro-Doppler signatures are reported.
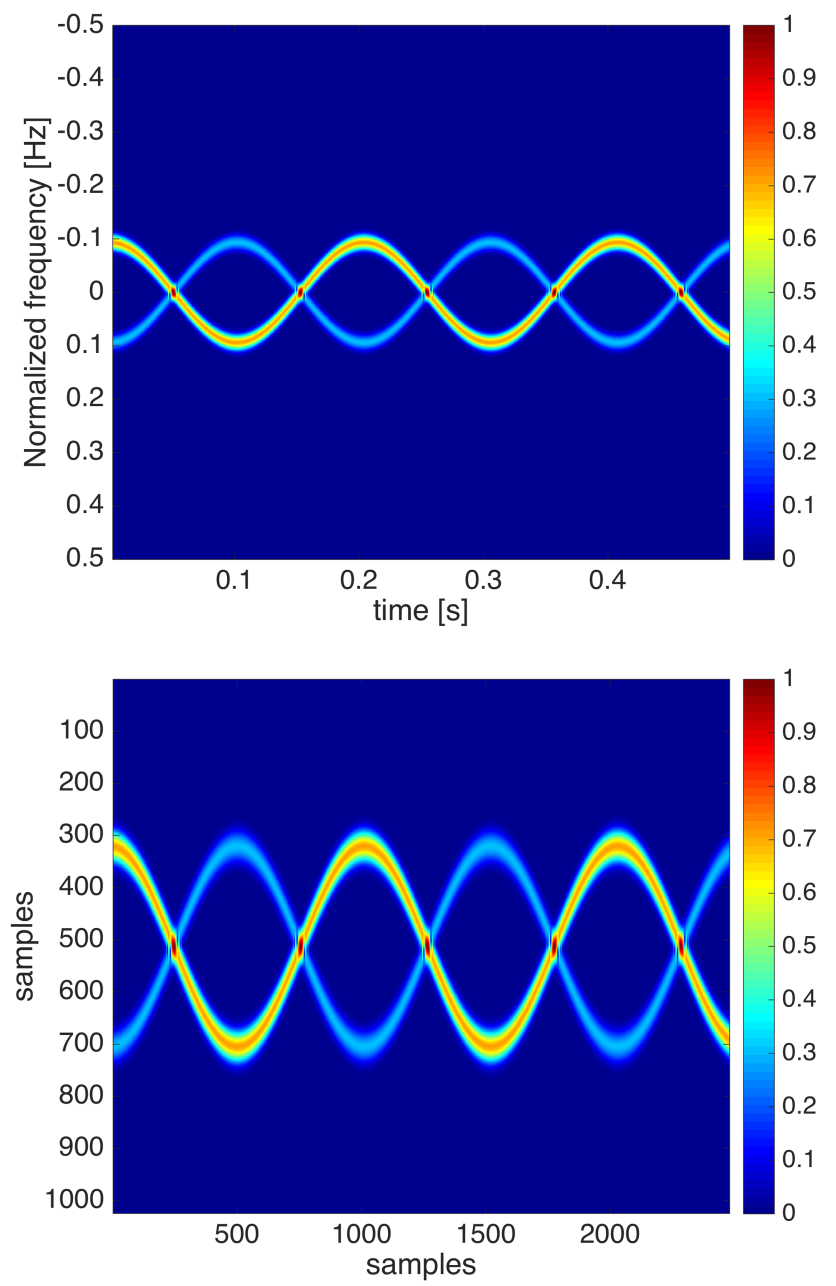

Fig. 5. Spectrograms of the signal received at the second receiver, before (top) and after (bottom) the re-sampling preprocessing. 
The received signals, $x_{1}(n)$ and $x_{2}(n)$, are generated on the basis of the model represented by Equation (1), in the case of $l=2$ and $M=2$. Both radar sensors have been simulated using a carrier frequency of $2.5 \mathrm{GHz}$ and a PRF of $20 \mathrm{kHz}$. The geometric scale factor is assumed to be 1 and 0.5 for the two receivers respectively. The target rotors has 2 blades of length $7.32 \mathrm{~m}$ and rotating at $4.9 \mathrm{rps}$. Finally the radar crosssections for each blade return are modeled as the following mixing matrix:

$$
\left[\begin{array}{ll}
\sigma_{11} & \sigma_{12} \\
\sigma_{21} & \sigma_{22}
\end{array}\right]=\left[\begin{array}{ll}
0.6 & 0.4 \\
0.7 & 0.3
\end{array}\right]
$$

as in a classical mixing problem.

All the steps illustrated in the above Section IV are applied, in accordance with the Block Diagran shown in Figure 3. The application of the proposed algorithm results in the extraction of the two generated components and the obtained results are presented in Figure 6 The Figure shows the unmixed sources after reshaping, in matrix form. Even if some residuals from the other source are still visible, the unmixing procedure effectively separates the two micro-Doppler contributions and allows to identify each of them separately.

\section{CONCLUSIONS AND FUTURE WORK}

In this paper the problem of micro-Doppler signature unmixing has been investigated. The proposed approach exploits the capability of ICA to separate hidden information in mixtures of observations. ICA has been applied to the spectrograms of received signals and the specific case of rotor blades return unmixing in a radar system has been analyzed. Simulated signals have been used to verify the performance of proposed technique. The results demonstrate the effectiveness of the proposed approach as an useful tool to unmix signatures, to be then exploited for advanced micro-Doppler based target recognition algorithms. Future work will validate the concept on real radar data, asses the radar cross section estimation capabilities and integrate the proposed approach in a target recognition algorithm. Additionally, the extension to the classification of miniature Unmanned Aerial Vehicles will also be considered by the authors.

\section{REFERENCES}

[1] S. Bjorklund, T. Johansson, and H. Petersson, "Evaluation of a microdoppler classification method on mm-wave data," May 2012, pp. 934 939.

[2] C. Clemente, L. Pallotta, J. J. S. A. De Maio, and A. Farina, "A novel algorithm for radar classification based on doppler characteristics exploiting orthogonal pseudo-zernike polynomials," IEEE Transactions on Aerospace and Electronic Systems, vol. 51, no. 1, pp. 417-430, Jan 2015.

[3] D. Gaglione, C. Clemente, F. Coutts, G. Li, and J. J. Soraghan, "Model-based sparse recovery method for automatic classification of helicopters," May 2015, pp. 1161-1165.

[4] K. Waheed and F. Salem, "Blind information-theoretic multiuser detection algorithms for DS-CDMA and WCDMA downlink systems," IEEE Trans. Neural Networks, vol. 16, no. 4, July 2005.

[5] J. F. Cardoso and A. Souloumiac, "Blind beamforming for nongaussian signals," IEE Proc. Radar Signal Proc., vol. 140, p. 362âĂŞ370, 1993.

[6] P. Addabbo, M. di Bisceglie, C. Galdi, and S. Ullo, "The Hyperspectral Unmixing of Trace-Gases from ESA SCIAMACHY reflectance Data," IEEE Geoscience and Remote Sensing Letters, vol. 12, no. 10, pp. 21302134, Oct. 2015.
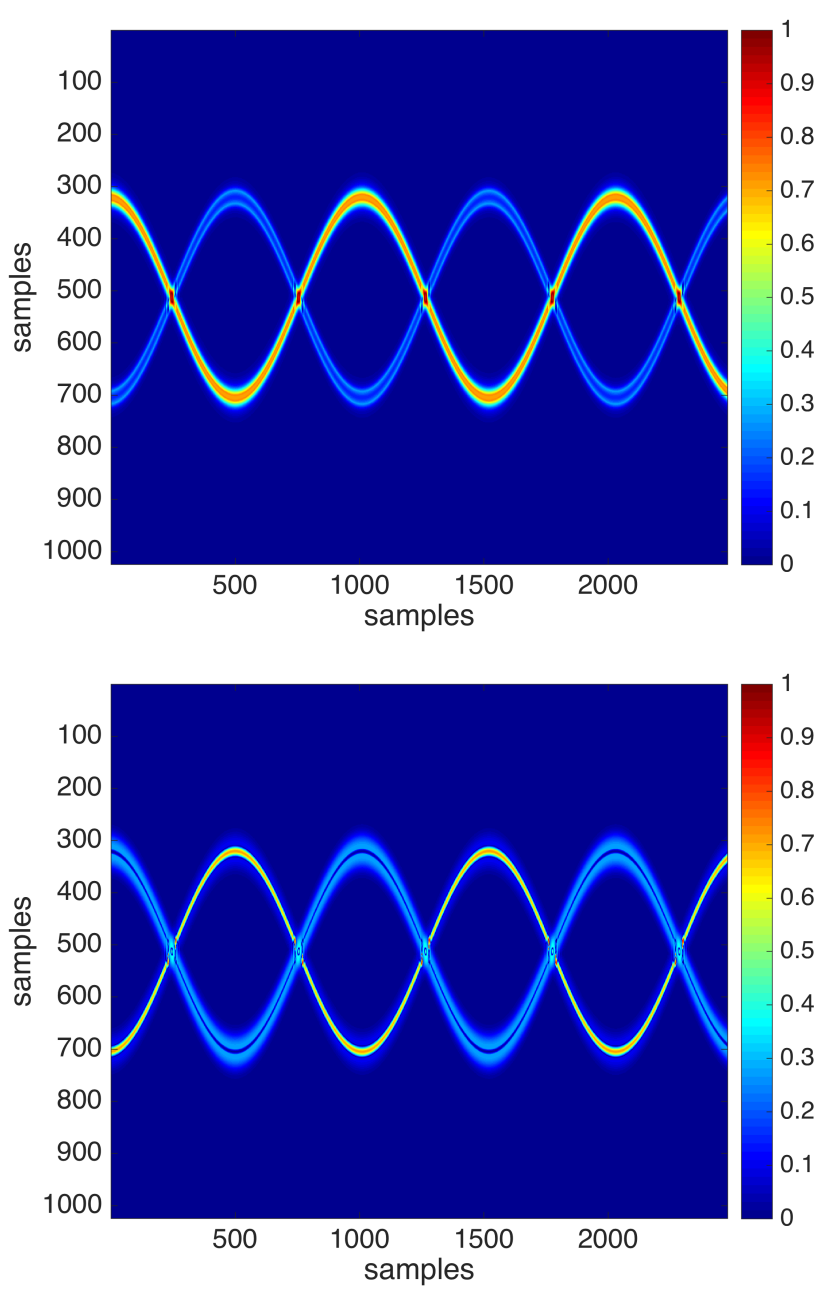

Fig. 6. The source matrices extracted by the unmixing procedure.

[7] V. D. Calhoun and T. Adali, "Unmixing fmri with independent component analysis," vol. 25, April 2006, pp. 79-90.

[8] J. Annemuller, T. J. Sejnowski, and S. Makeig, "Complex spectral domain independent component analysis of electrocephalographic data," March 2003.

[9] M. Novey and T. Adali, "Using complex-valued ICA to efficiently combine radar polarimetric data for target detection," April 2009, pp. $1673-1676$

[10] V. C. Chen, "Spatial and temporal independent component analysis of micro-doppler features," May 2005, pp. 348-353.

[11] V. Chen, "Analysis of radar micro-doppler with time-frequency transform," 2000, pp. 463-466.

[12] E. G. Learned-Miller and J. W. Fisher, "Ica using spacing estimates of entropy," vol. 4, Dec 2003, pp. 1271-1295.

[13] M. van der Baan, "Pp/ps wavefield separation by independent component analysis," vol. 166, no. 1, Jul 2006, pp. 339-348.

[14] T. Cover and J. Thomas, Elements of Information Theory. Wiley, 2006.

[15] P. Addabbo, M. di Bisceglie, and C. Galdi, "The unmixing of atmo2012 . 